AperTO - Archivio Istituzionale Open Access dell'Università di Torino

\title{
Design and Development of a Social, Educational and Affective Robot
}

\section{This is the author's manuscript}

Original Citation:

Availability:

This version is available http://hdl.handle.net/2318/1742267

since 2020-06-25T12:28:06Z

Publisher:

IEEE

Published version:

DOI:10.1109/EAIS48028.2020.9122778

Terms of use:

Open Access

Anyone can freely access the full text of works made available as "Open Access". Works made available under a Creative Commons license can be used according to the terms and conditions of said license. Use of all other works requires consent of the right holder (author or publisher) if not exempted from copyright protection by the applicable law. 


\section{Design and Development of a Social, Educational and Affective Robot}

\author{
Cristina Gena \\ Department of Computer Science \\ University of Turin \\ Turin, Italy \\ cristina.gena@unito.it
}

\author{
Massimo Trainito \\ School of Communication Science \\ University of Turin \\ Turin, Italy \\ massimo.trainito@edu.unito.it
}

\author{
Claudio Mattutino \\ Department of Computer Science \\ University of Turin \\ Turin, Italy \\ claudio.mattutino@unito.it
}

\author{
Chiara Vaudano \\ Department of Computer Science \\ University of Turin \\ Turin, Italy \\ chiara.vaudano708@edu.unito.it
}

\author{
Gianluca Perosino \\ Department of Computer Science \\ University of Turin \\ Turin, Italy \\ gianluca.perosino@edu.unito.it \\ Davide Cellie \\ School of ICT \\ University of Turin \\ Turin, Italy \\ davide.cellie@edu.unito.it
}

\begin{abstract}
In this paper we describe the approach and the initial results obtained in the design and implementation of a social and educational robot called Wolly. We involved kids as co-designer helping us in shaping form and behavior of the robot, then we proceeded with the design and implementation of the hardware and software components, characterizing the robot with interactive, adaptive and affective features.

Index Terms-educational robotics, human robot interaction, adaptive systems
\end{abstract}

\section{INTRODUCTION}

The term educational robotics [1], [2] refers to the use and development of learning environments based on robotic technology, mainly robots and software capable of programming them. In this field the kind of robots may include toy robots, articulated robots, mobile robots, and more. Educational robotics can be suitable for students across the entire educational spectrum, from primary to university students.

Educational robots may play different role as helping children in learning basic algorithms by programming robots and their actions; supporting learning, such as in case of simulation of actions and phenomena; allowing children to participate in class sessions by taking control of a remote-controlled robot, for distance learning when students cannot participate to the lessons; assisting teachers in their daily tasks through a collaborative, complementary human-robot approach, etc.

In the last years there has been an increasing interest for social robots in education [21]. According to Breazeal [22] a social robot is an autonomous robot that interacts with people by engaging in social-emotive behaviors, skills, capacities, and rules attached to its collaborative role. For Fong [23] social robots are able engage in social interactions, they possess histories (perceive and interpret the world in terms of their own experience), and they explicitly communicate with and learn from each other. To reach these goals, in the field of

This work has been partially funded by the 2019 Google Educator PD Grant. social and educational robotics, there has been a growing interest for adaptation mechanisms able to tailor the interaction with the final user/student [24] also in a long-term interaction perspective. Preliminary results showed that adaptation might help in maintaining the user engagement also when the initial "wow" effect disappears [21].

There is a general consensus in learning literature about the positive effect of a social and playful interaction in children learning [25], [26] joined to the importance of having a competent peer guiding the learning process. In this sense learning activities with educational robots perfectly fit these requirements. In addiction to that Ahmad et al. [21] have shown that appropriate and emphatic feedback has a positive effect on child-robot interactions especially in a long-term perspective.

Believing in the beneficial role of social and educational robotics to support learning, in our HCI lab we have codesigned with students and then realized a social educational robot [4]. The robot is made of a very common hobby robotic kit, is able to move through its four independent motorized wheels, and can be controlled trough a web app. Its body has been almost completely 3D printed. The head of the robot is made of an Android-based smartphone, hosted on servomoter, and able to show and understand emotion, and to speak and to understand voice command. The main goal of the robot is acting as an affective peer-tutor for children: it is able to execute a standard set of commands, compatible to the ones used while coding, but also to interact both verbally and affectively with students about their results, and in the future it will adapt its behavior depending on the user's features, the context and the perceived user emotions. Thus as longterm goal we plan to enrich the robot with a user modeling component, which will keep track of past interactions with the user, will reason about her on the basis of her features, her skills, and her emotions recorded during past interactions.

This paper has been organized as follows: Section II 
presents the state of art, Section III introduces background and motivations, Section IV presents the co-design and then the realization of the first prototype, Section V presents the robot main interactive features, Section VI describes an initial experimentation and its results, and finally Section VII concludes the paper and presents future work.

\section{STATE OF THE ART}

Educational robotics originates from the constructionism of Saymour Papert [15], which originated in turn from the theories of constructivism by Jean Piaget [16]. For Papert the construction of knowledge is more significant in a context where the learner is engaged in the realization of something concrete, tangible and shareable.

An educational robot is a perfect example of the Papert's cognitive artifact, definition that involves objects and devices that facilitate the development of specific learning capabilities. Educational robotics combines theory and practice through manual and playful exercises, allowing students to develop personalized learning processes and strategies.

According to Paiva et al. [14] in order to to have successful human-robot interactions, robots must be endowed with emotional processing, as well as be able to respond emotionally to the humans and adapt to their environmental and moral context. For Leite et al. [10] results indicate that robots are well accepted in educational settings, and that mechanisms that promote social and emotional interactions between robots and children contribute to a great extent in this acceptance. Regarding pupils' age, younger children are more likely to engage with robots, possibly because they do not have any preconceptions of what a robot is supposed to behave, and they may see it simply as a very special/advanced kind of toy.

Another key point for a successful interaction between child and educational robots relies in their proposed role. Kanda at al. [9] first proposed the idea of robots as peer tutor for children. According to the authors robots could form relationships with children and children might learn from robots as they learn from other children if they may found a common ground to establish a social relationship, such as recognizing the other persons identity, discovering similarities, etc. All of them are key issues for cementing a social relationship, which has then to evolve along time, as in case of human-to-human relationship.

Ahmad et al. [21] present an emotion and memory model for a social robot and the robot then adapted its behaviour based on the developed memory. The model was applied on the NAO robot to teach vocabulary to children in a playful setting, and the authors conducted a set of exploratory evaluations. Preliminary results showed that the so generated robot behaviour was able to sustain social engagement after the first interaction, in a long term interaction perspective. In addition, it also helped children to improve their vocabulary. Results also showed that the condition where the robot displayed positive emotional feedback had a significantly positive effect on the childs vocabulary learning performance as compared to the two other conditions (negative and neutral feedback).

\section{BACKGROUND}

During the academic year 2017-18 we carried out a set of coding activities, which lasted about 3 months, in a third year primary school. These activities were aimed to teach students not only the basic of programming, but also a new language and a new way of thinking and solving problems: what is called computational thinking. Computational thinking is commonly defined as a set of attitudes and skills that make people face and solve problems in a way that takes inspiration from computer-science: that is, to make people think as a computer scientist [27]. In order to reach these goals, we structured our lessons following the program of the Course 2 from Code.org ${ }^{1}$, with some variation described below. In particular we focus on the following topics: Sequences, Cycles and Repetitions, Conditional instructions, Events. During the execution of coding exercises we noticed that children encountered some difficulty, mostly related to orienteering problems: for instance it was still difficult for them to recognize the left and the right side, especially when the virtual robot on the screen was not turned from their point of view. Thus in order to strengthen their sense of orientation and give them greater security in spatial orientation, we alternate classical plugged coding activities with unplugged games (e.g. instructing a child to move on a floor chessboard) and also with educational robots like Clementoni DOC ${ }^{2}$, Dash $^{3}$, MakeBlock ${ }^{4}$, which may be programmed using coding instructions. By observing the children, we believe that in particular these last activities have contributed to a better understanding of spatial and orienteering concepts. Before starting the coding course and at the end of the final lesson, we distributed two questionnaires developed by La Sapienza University ${ }^{5}$ to each student, with the aim of testing their knowledge and skills before and after coding activities. Each of these consisted of five questions requiring generic skills such as spatial orientation on a two-dimensional plane (Geography questionnaire) and basic knowledge in geometry (Geometry questionnaire). The questions in the final questionnaires, although differently prepared, had the same degree of difficulty as the first ones.

At the end of the course, we analyzed and compared the scores obtained by each child before and after the coding experience. Regarding the questionnaire on Geography/Orienteering we calculated the average value in pre- and post-test: 2.28 ( $\mathrm{SD}=0.23)$ vs $3.04(\mathrm{SD}=0.25)$. By comparing the values by means of a dependent T-test, $t(24)=-2.854, p$ $<0.009$, we conclude that there was a statistically significant improvement between the scores collected before and the ones collected after the course, witnessing that the coding lessons, enriched by those ones of educational robotics, have had a positive effect on children spatial orientation capability, which significantly improved.

\footnotetext{
${ }^{1}$ https://studio.code.org/s/course2

${ }^{2} \mathrm{http} / / / \mathrm{www} . c l e m e n t o n i . c o m / i t / 11112$-doc-robottino-educativo-parlante/

${ }^{3}$ https: //www.makewonder.com/dash/

${ }^{4}$ https://www.makeblock.com/steam-kits/mbot

${ }^{5} \mathrm{https}: / / \mathrm{www} . c l e m e n t o n i . c o m /$ static/front/areadocenti/docs/Questionari.zip
} 
Regarding the scores obtained on Geometry, we calculated again the average values in pre- and post-test: $3.6(\mathrm{SD}=1.58) \mathrm{vs}$ $4.0(\mathrm{SD}=1.15)$, which resulted higher with respect to the Geography ones, suggesting that kids had a stronger background in Geometry since the beginning. By comparing the values by means of a dependent T-test, $t(24)=-1.188, p<0.246$, we conclude that there was not statistical difference between preand post-test, witnessing that the coding exercises produced a lighter improvement in Geometry, with respect to the one registered in Geography, but since the scholars started with a higher background (Geometry was part of their program) that was slightly improved after our lessons.

At the end of the coding activities, we have also distributed a survey to the children, in order to collect information about their satisfaction and engagement. The survey consisted of 15 questions in which children can respond by giving assessments included in a scale of five values expressed thanks to a Smileyometer [18], which is the most used tool for the measurement of children's opinion and includes an evaluation scales through the smileys corresponding to a range from 1 to 5 (from strongly disagree to strongly agree). The children were asked to express their opinion by choosing one of the faces. The survey was anonymous, in order to protect children's privacy.

The feedback obtained allowed us to conclude that the lessons have been appreciated and have produced satisfactory results; more than $68 \%$ of the judgments obtained the strongly agree level of satisfaction and $17 \%$ obtained an agree, with $84 \%$ of children that strongly agreed on finding Interesting the proposed activities and a $92 \%$ that strongly agreed that they would like to Have more lessons of this type.

At the end of these three months of positive experience, we realized that having a robot that could perform the same actions as code.org virtual robots perform, could be a good solution for children, especially in order to help them solve orientation problems. We cannot exactly conclude from the statistics whether the data relating to the children improvement in orienteering are due only to the use of robots, but from our observations in the field we have noticed the benefits brought by the presence of a cognitive artifact, such as the robot is.

Therefore, seeing that no commercial robots has the features we would like, we decided to create an educational robot on our own, equipped with social, interactive and affective capabilities, able to involve children and to establish a social and emotional bond with them in order to increase their learning experience and their engagement. We decided to design the robot as open source project, made with a low-cost kit that could be easily reproduced and improved by anyone who wanted it.

\section{THE ROBOT DESIGN}

As described in details in [4], before realizing the robot, we conducted a co-design session with 25 children. The results of our inductive analysis suggested that the main designed requirements should be: The robot should be a child professor/assistant able to teach and play in a funny way; It should express its emotions through its voice, its cartoon-like face (e.g. big eyes and smiley face, etc. ) and its luminous physical parts; It should be gender-neutral; Its body must be a little bit squared; It should have playful and colorful dresses/accessories as a jacket and a papillon; should have super powers, such as heating, cooling, measuring fever, etc.

Following indications drawn from the co-design process, we designed the appearance and structure of the robot (see Fig. 1) as will be described in the following.

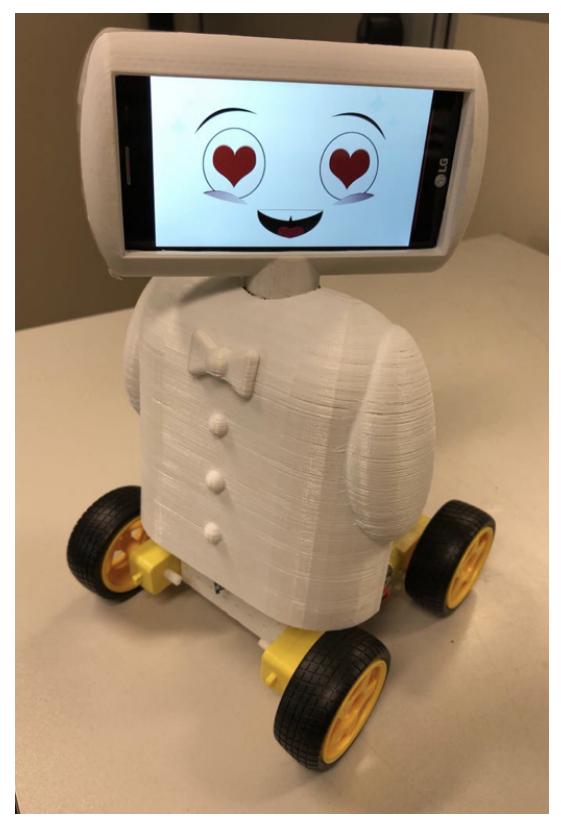

Fig. 1. Wolly, the educational robot

The body has been designed as simple and squared, in line with the children imagination, but with rounded and softened edges, more suitable for a toy designed for primary school children. The arms, although not working, have been added to meet the expectations of children, and to favor the emergence of automatic mechanisms of anthropomorphization, essential to establish social relationship. An internal skeleton, joining the motorized base with the head, constitutes the central structural part, protects and supports the electronic boards and it is prepared for future addition of new sensors or gadgets. The control logic of motorized components has been implemented in an Arduino Mega 2560 card, which interprets the commands to be executed via USB or serial to 115200 baud. It is connected to an Adafruit Motor Shield V1.2 motor driver, for communication with the 4 3-6 Volt DC motors, on which depends the wheel movement. A Servo MG995 of 5V DC and $400 \mathrm{~mA}$, tied to the head, allows small head rotations and helps to simulate an attentive behavior. A $6800 \mathrm{mAh}$ Powerbank LiPo battery with 5V 1A and 5V 500mA regulated outputs offers power to all the electronic cards. Finally a Wemos D1 mini card provides wireless communication with the robot, offers server functionalities and exposes REST APIs that provide a set of basic instructions (e.g., moveForward, turnLeft, turnRight, moveBackward, etc.) for controlling the 
motors and the servo. The head consists of a $1 \mathrm{GHz}$ quad core LG Smartphone, with 1.5GB of RAM and 16GB of ROM, running Android 7.0. The interaction with all the functions of movements, listening, speech and emotion management is implemented in a set of Android apps running on the smartphone and dialoguing also with external web services, and able to control the engines through the REST APIs.

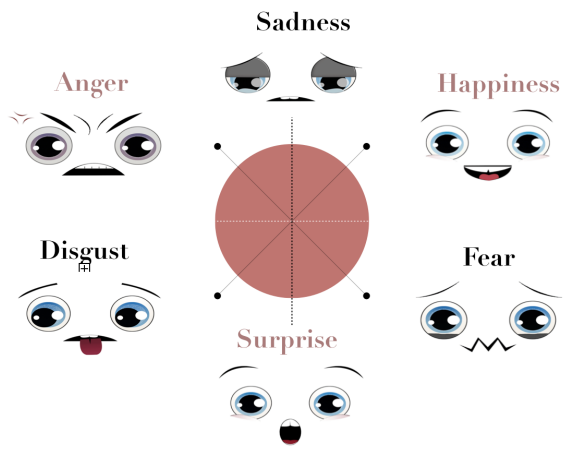

Fig. 2. The six basic emotions expressed by Wolly

As far as the face of the robot and its expression are concerned, we used the Android-based smartphone as a screen in which to show the robot's face and its the expressions and emotions. An Android application, called AffactiveApp, displays an animation of the face of the robot (namely an animated GIF with blinking eyelids), expressing one of the six basic emotions identified by Ekman [6] (happiness, fear, surprise, disgust, anger, sadness, see Fig. 2) plus a neutral one, depending on the context and on the identified user's emotion.

The final result has been the one depicted in Figure 1: a little robot with childlike features, also reproduced in the mimicry of facial expressions and in the chosen voice, with autonomy of movement and social skills of recognition and expression of simple emotions. The body has been printed with semitransparent plastic material, to allow the light coming from the LEDs, which could be inserted inside so as to allow the diffusion of light. Buttons and bow ties have been added for giving the robot a playful and elegant appearance, as suggested by kids.

\section{THE Robot InTERACTIVE BEHAVIOR}

As introduce before, the main goal of the robot is acting as an affective peer-tutor for children: being able to to execute a standard set of coding commands, to interact both verbally and affectively with students about their exercises and final results, and finally adapting its behavior depending on the user's features, the context and the perceived user emotions. As longterm goal we plan to enrich the robot with a user modeling component, which will keep track of past interactions with the user, will reason about her on the basis of her features, her skills, and her emotions recorded during past interactions. As short-term goal, we leveraged the main role as a social educational and affective robot targeted at helping kids in coding exercises. Thus the first release has been targeted at having the robot able to give the children the main instructions on how to reach their goal while doing the exercise and how write the code (by assembling visual blocks), and at the same being able to execute the given instructions, i.e. moving on a chessboard and reaching the given goals, and finally interacting with kids in a basic affective way, i.e. being able to recognize kid emotions and feeling and to react accordingly. The design has been declined in the creation of a cognitive artifact that has to be not exclusively an adaptable or programmable tool to be used in support of teaching: rather, such artifact should have sufficient memory and autonomy to be able to help, execute and interact with children, and being able to experience the effectiveness of its social functions.

In order to reach these goals, also inspired by the codesign session described in Sec. IV, we thought of a first set of essential requirements, but others could be added in the subsequent phases of design:

1) Having motorized and measurable movements on wheels and rotation of the servo connected to the head either autonomously or remotely controllable;

2) Making 90 degree rotations in each direction, thus being able to make movements on the cells of a pre-defined grid, such as a chessboard, in order to be able to execute typical coding exercises (e.g. move left/right and go forward and backward);

3) Being programmable in its functions, such as movement and behavior, through both a visual interface and visual commands (e.g. Blockly ${ }^{6}$ ) from remote devices, and a voice interface in direct interaction with the robot, or a combination of the two modalities;

4) Receiving, processing and understanding voice commands according to a communication protocol that simulates a natural dialogue; being able to verbally communicate in accordance, i.e. being able to convert a written text into speech even at the time of execution (verbal communication);

5) Expressing emotions by showing a digital representation on the display (i.e. facial animation), or by means of lights (the internal the luminous LEDs) and servo movements (non-verbal communication);

6) Recognizing the emotions of the users, with a consequent ability to adapt and diversify the emotional/affective response on the basis of the emotion perceived (emotional intelligence);

7) Directing the conversation by making use of contextual information, in a continuous exchange of stimuli and responses that are dynamic and adaptive with respect to the environment;

8) Understanding and evaluating the correctness of coding exercises, recognize errors and propose solution strategies when required.

We have made these requirements converge into a first release of a software architecture that controls the main components (natural language interaction, affective interaction,

\footnotetext{
${ }^{6}$ https://developers.google.com/blockly/
} 
movement controls) puts them in communication with each other. For the development of the interaction strategy, the behavior and the memory settings of the robot, we have borrowed a concept from cognitive psychology: the script [17]. A script refers to a collection of standardized actions, performed individually or in relational contexts, suitable for forming and consolidating mental schemes. It consists of a set of actions carried out in order to achieve a well-defined purpose which, through repetition over time and learning through experience, are abstracted into a more general model and recorded in procedural memory as a sort of unconscious scripts to be called at the right time. During learning and knowledge building processes, scripts become more complex and elaborated, being able to request, for the satisfaction of the intended goal, the activation of a set of sub-scripts and a set of sub-targets. Scripts are very similar to the Marvin Minsky's concept of frame [12], namely an intelligence data structure used to divide knowledge into substructures by representing stereotypical situations. Frames are between the most used data structure in artificial intelligence and in the natural language processing field.

In the design of Wolly's abilities, we applied a formalized representation of the script model, intending the script as a specific interaction containing a dynamic collection of events, to be reproduced as a sequence that can be adapted to the contextual situation. To this end, a sets of script encoding domain objects have been defined, and also a player able to executing them. The addition to of new features and behaviors could consist not necessarily in writing new scripts, but also in assembling existing ones. In Figure 3 it is possible to observe a simplified schematization of the architecture, which will be discussed in the following.

A script must contain a sequence of actions that the robot should perform during a specific interaction scenario, and each action must correspond to one of the functionality established in the requirement specifications: it could be either the execution of a movement, or the expression of an emotion, or a sentence in natural language, for instance. The intended sequence is not static but can be dynamically determined on the basis of the data collected during the interaction itself. To guarantee these properties, we decided to represent a script as a graph of interaction blocks, whose arcs and linked nodes can be dynamically modified at runtime (see Fig. 3). Each block may contain the actions to be executed by the robot, and specifies its successor node, eventually updatable during the interaction.

Looking at Figure 3, we may notice that, in particular, the InteractionScript object preserves the references to the first block of the script or to the one currently running, while the InteractionScriptPlayer (the player) is in charge of the script execution: starting from the first node it executes all the specified instructions, and then moves on to the next one following the order dynamically established from the arcs, and reiterating the procedure until the blocks are exhausted. Each block can be programmed simply by specifying the components that the programmer wants to use for the defined sub-action, while the player will take care of their execution and management. These components can be: The facial expression to be shown on the display during the execution of the block, represented by the object Emote; A set of Textual variants to be turned into speech; An InteractionCommand object containing the definition of any action that is not a speech or an expression, e,g, a movement; The Pause that must elapse between the execution of a block and the next one; The Successor block. The choice to use a set of textual variants at block level, instead of a single simple text string, allows to create a simple mechanism of simulated intelligence: the player takes care of randomly extracting a variant from the provided list and then it turns the variant into speech, thus avoiding that any new execution of the script is repeated as to the previous one (a similar approach is also adopted by the commercial Pepper $\left.\operatorname{robot}^{7}\right)$. The variants must be consistent with each other and freely interchangeable, differing only in their form and not in their content. This choice has been done in order to favor anthropomorphization mechanism as it is a strategy to weaken somehow the perception of an agent (the robot) with a static and predetermined behavior. The condition of interchangeability is however restricted as the system provides a further possibility linked to the emotional intelligence which the robot is equipped with: each text variant added to the block can be tagged with a reference to a particular emotion, represented in the diagram by the Emotion object. Since the player is integrated with an emotion recognizer, is able to update itself at the block level on the last emotion detected in the interlocutor, and therefore it will randomly select a textual variant only among those ones fitting the recognized emotional state. This means that the interchangeability of the variants must be respected only among those ones associated to the same emotional tag (e.g. happiness, surprise, sadness, etc.). The system provides a dual way to manage the Wolly's emotional intelligence, at:

- Script level: by setting up dedicated blocks in response to the detection of specific emotional states, and directing the execution flow to those blocks at runtime. This choice is suggested when the management of a particular emotional state requires more than one block;

- Block level: merely labeling the variants and characterizing them in the more appropriate way. This choice simplifies the approach and limits the branches of the script graph, when a single block is sufficient for the emotional state management.

The introduction of this approach has highlighted a new requirement: selecting a facial expression to be displayed on the display only at block level is not sufficient. Since the presence of textual variants to be provided in response to different emotions is expected, it is necessary that the relative expression also conforms to the different emotional state. It has been therefore decided to introduce a SpeechVariant object for the representation of textual variants, able to specify an expression or a pause even at the variant level. When an

\footnotetext{
${ }^{7}$ https://www.softbankrobotics.com/emea/en/pepper
} 


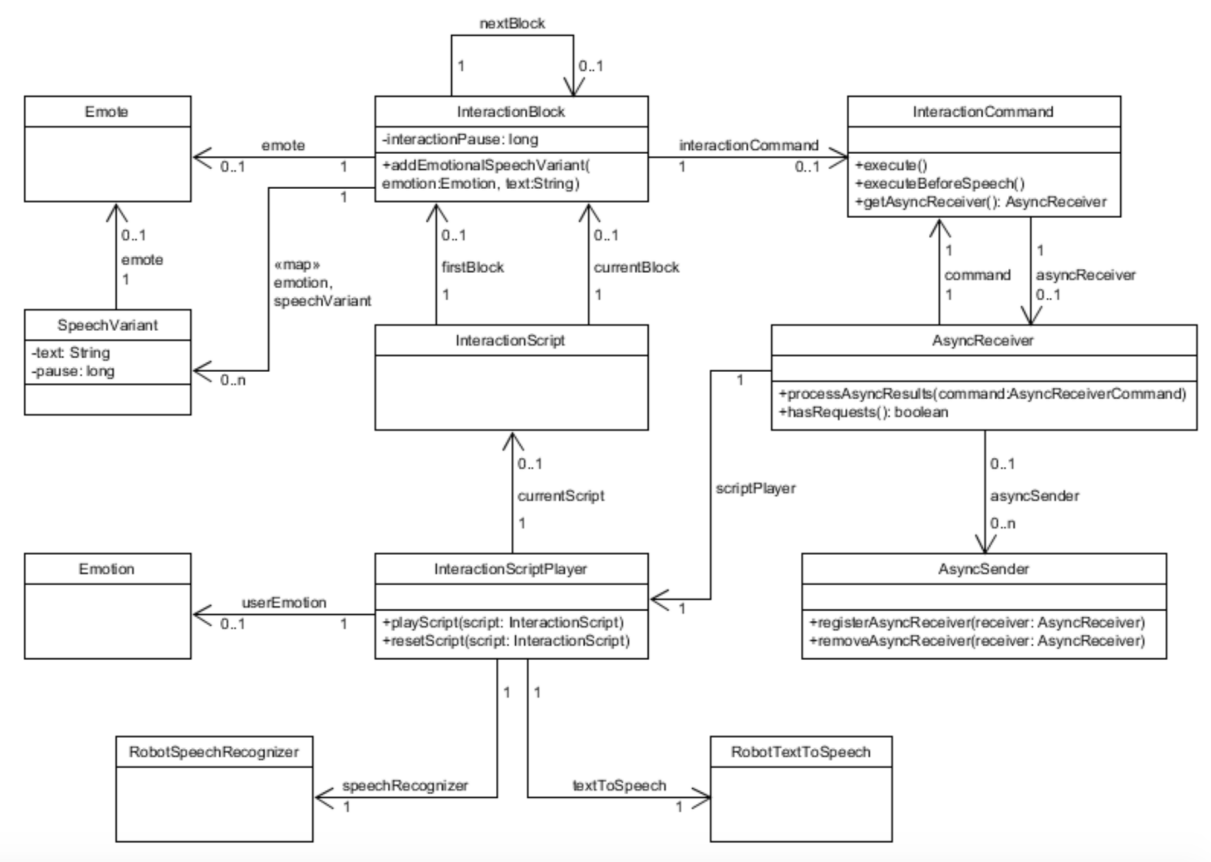

Fig. 3. Portion of the high level architecture of the script system.

expression or a pause is specified at block level, it could be overwritten when a more specific variant is required.

Anogther significant component of the interaction system is represented by the InteractionCommand object which, thanks to an asynchronous call collector, allows the block-level definition of any type of action other than the expression of an emotion or a voice reproduction. This object provides a sets of bodyless methods that each block must redefine with the the envisaged actions, then the player will take care of their invocation at the right time. The methods provided are as follows:

- execute(): for the definition of standard actions that do not have particular preconditions about their starting time during reproduction of a single block; this method is invoked by the player only after the extraction and execution of the textual variants, and after respecting any specified pauses;

- executeBeforeSpeech(): for the definition of actions that the robot might perform before the speech phase, for example for setting variables;

- processAsyncResults(): for the definition of actions whose execution requires the preliminary acquisition of data in real time, as could be the signal of the end of the listening or movement phase.

Within these methods it is possible to program any type of functionality, and the player will take care of the management of the events related to the TextToSpeech libraries to detect the beginning and the end of the speech phase and then invoke them as soon as possible.

The native Android libraries, providing text-to-speech ${ }^{8}$ and

\footnotetext{
${ }^{8} \mathrm{https} / / /$ developer.android.com/reference/android/speech/tts/TextToSpeech
}

speech recognizer ${ }^{9}$ functionalities, have been used for playing the Wolly's voice and recognizing the spoken words. The ability of these libraries to process the text at runtime has been very useful, providing Wolly with the possibility of enriching its spoken word with variables whose value can be determined during the execution of the script. At the moment the robot's speech recognition abilities are limited to a set of keywords that it is able to recognize, e.g. in response to its questions or in relation to general questions that it is able to manage (about itself and its inventors, etc.). The emotion recognition software (AffectiveApp) is based on Affectiva software ${ }^{10}$. The camera connected to the smartphone (Robot's face) acquires frames and feeds them directly to the facial expression engine connected to Affectiva that answer, in turn, the detected emotion and their associated certainty level (the emotion with higher value is the one suggested by Affectiva). We have implemented and tested a similar approach in other works [20] obtaining results consistent with the real emotion expreassed by the user.

\section{A GAME-BASED EXPERIMENT}

Before starting with the integration of the coding exercises and the robot adaption based on the user detected emotion, we decided to implement a game stimulating the interaction between the kids and the robot.

Thanks script-based approach described above, it has been possible to set up a a word guessing game (inspired to the famous Taboo game), characterized by a sets of riddles that the robot exposes for the identification of a word it is thinking of. The game has been implemented as follows:

\footnotetext{
${ }^{9} \mathrm{https} / / /$ developer.android.com/reference/android/speech/SpeechRecognizer ${ }^{10} \mathrm{https}: / /$ www.affectiva.com/
} 


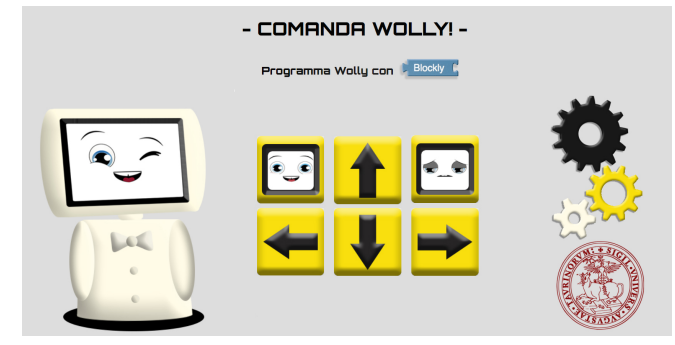

Fig. 4. The simplified web-based interface.

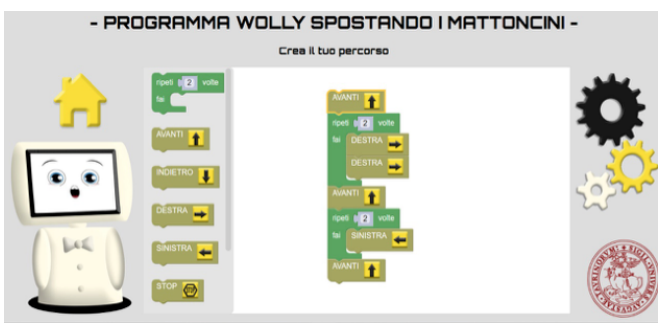

Fig. 5. The Blockly-based web interface.

1) The game starts with the robot that presents itself (and also asks the children if they have any questions about it) and then explains the game rules;

2) The robot provides the first clue and lets the children think about twenty seconds;

3) After 20 seconds the robot emits a sound;

4) Children say a word (children can be divided into teams, all together or one alone);

5) If the word is correct, the robot compliments and is happy, and then it will ask if they want to play again;

6) If the word is wrong, the robot says to try again and provides another clue. It continues up to a maximum of 3 or 4 clues (the clues are always more intuitive, with the last one extremely obvious);

7) If children do not guess the right word, the robot will be sad and cheer them up by telling to try again. He will also pronounce the word not found.

According to the possible results, Wolly will show the following emotions according to these rules (see Fig. 1):

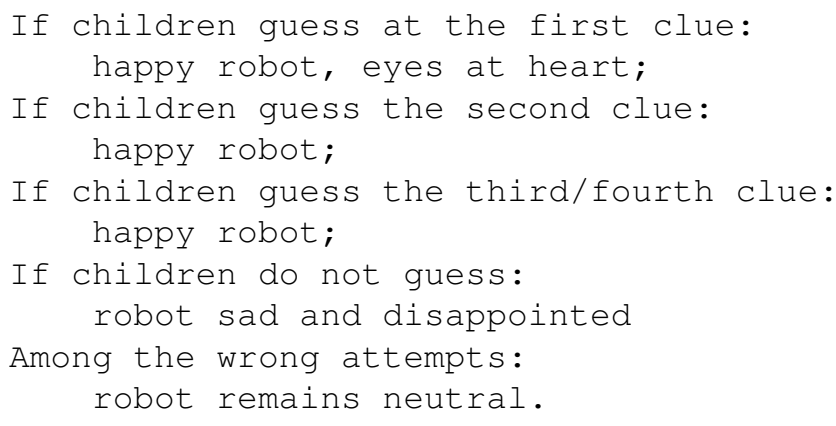

We experimented the Taboo game in two field trials (which are considered essential for the development of Human Robot Interaction, as suggested in [9]): the first one with the same class that helped us in the co-design (third grade of primary school), and also with older fifth grade children. The trials lasted less then one hour, however allowed us to formulate the following qualitative observations:

- Children were excited about playing with a robot. It was a nice example of an educational robotics event, that is, an exchange of communications between them and the robot. Through the game, children understand how to give the correct instructions, e.g. speaking clearly and slowly;

- Children very amused by being all together or in different teams and having to reflect and find the solution all together (team work);

- Waiting times between the robot request and the children reply were correct (15-20 seconds). Even if, however, when the words are simpler, the time could be dynamically reduced;

- During the first attempts it was difficult to explain children that they had to wait for the sound signal before giving the answer, but after a while they understood how to take turn;

- Children were instructed to make little noise and this gave them a sense of responsibility;

- When children found the word they were overjoyed and jumped;

- Children were struck and admired Wolly, and its capability of showing different expression and talking. Those more experienced and passionate also asked for more technical details (hardware and software) about it.

\section{CONCLUSION AND FUTURE WORK}

In this paper we have presented our experience with Wolly, an educational robot able to perform emotion recognition and affective and natural language interaction. Our approach needs to be extended in order to improve those functionalities: Wolly has a very limited but meaningful speech recognition vocabulary, useful just for a simple dialogue management approach. Its emotion recognition ability shows good performance in a set of test we have performed in our lab, but it is now limited to emotion detection functionality of Affectiva libraries.

Future developments will try to overcome the above limitations, and we are also leveraging the movement capability of the robot, in order to create interactive paths fitting the coding excise requirements. We are also expanding the script approach in order to extend the knowledge base and the behavior of the robot, and giving it a well-defined personality, as a way to entice and foster effective interaction as suggested by Fong et al. [23]. As long-term goal, we would like to use educational robotics also for children with special needs, and help them to learn coding in an interactive way. In order to achieve these goals and also the improve the effects of the long-term interaction with the robot, we will also integrate into the robot software architecture a user modeling component (similar to the one described in [20]) able to adapt the interaction according to the user features and needs, and also her past interactions and progresses.

As far as short-term goals are concerned, we are now implementing a set of lessons to be executed by the robot, 
following the Course 1 and 2 of code.org ${ }^{11}$, and deploying a cloud-based architecture to manage its natural language and affective-based interaction, as described above. Our goal is having Wolly as coding-helper and performer, in an interactive and affective way, thus being able to modify its behavior according to the emotion detected in the child working with it. We are also working for integrating Blockly ${ }^{12}$ to the web-based command interface, and we have just made some advancement by customizing Blockly for the Wolly's ability to be programmed in a coding-like modality. As you can see in Figure 5, in the left column, called "toolbox", there are bricks presenting the main ability of Wolly (e.g. movements), with the exception of the green one, which the user can use to insert the blocks inside who wants them to be repeated as many times as desired; just change the number inside. The white area, on the other hand, is the "workspace" in which the blocks can be dragged, thus forming our puzzle; that will be the set of instructions that the robot interpreter will transform into executable code.

We are also testing a personalized visual set of instructions for giving basic commands to Wolly (move, speak, talk, etc.). In fact, we have performed a set of experiments involving again kids as co-designers in order to define with them set of commands to control the robot's actions and behavior [3]. The co-design helped us in designing a simplified web-based interface, made up of buttons that allow the robot to execute commands and express basic emotions as if it were managed by a remote control (see Figure 4 ). The commands are executed one at a time, for example the "forward" arrow will move the robot on right direction for about two seconds, and the user can select another command. We evaluated in the wild this basic interface during a robotics fair near Turin ${ }^{13}$. Several children and their families tried the proposed commands and they were able to use them without problem. Notice that this interface is targeted to pre-scholar user and/or for designing the behavior of Wolly, as if it were a social robot.

\section{ACKNOWLEDGMENT}

We would like to thanks all the students the help us in the co-design experiment. We would like also to thanks the 4A class of Falletti Primary School, Turin and their teachers Linda Garofano and Teresa Carretta, and Valerio Cietto for the first release of Wolly, and Mauro Giraudo for the 3D printing.

\section{REFERENCES}

[1] Dimitris Alimisis, Educational robotics: Open questions and new challenges, Themes in Science and Technology Education 6 (1), 63-71

[2] Fabiane Barreto Vavassori Benitti, Exploring the educational potential of robotics in schools: A systematic review, Computers \& Education, Volume 58, Issue 3, 2012, Pages 978-988, ISSN 0360-1315, https://doi.org/10.1016/j.compedu.2011.10.006.

[3] Gianluca Bova, Davide Cellie, Cristina Gioia, Fabiana Vernero, Claudio Mattutino, Cristina Gena:End-User Development for the Wolly Robot. IS-EUD 2019: 221-224

\footnotetext{
${ }^{11} \mathrm{https} / / /$ studio.code.org/s/course1, https://studio.code.org/s/course2

${ }^{12} \mathrm{https} / / / \mathrm{developers.google.com/blockly}$

${ }^{13} \mathrm{https}: / / \mathrm{www}$. robotikavallauri.it/eventi
}

[4] Valerio Cietto, Cristina Gena, Ilaria Lombardi, Claudio Mattutino, Chiara Vaudano: Co-designing with kids an educational robot. ARSO 2018: $139-140$

[5] Eguchi, A. (2010). What is educational robotics? Theories behind it and practical implementation. In D. Gibson B. Dodge (eds.), Proceedings of Society for Information Technology and Teacher Education International Conference 2010 (pp. 4006-4014). Chesapeake, VA: AACE.

[6] Ekman, Paul (1999), Basic Emotions, in Dalgleish, T; Power, M, Handbook of Cognition and Emotion (PDF), Sussex, UK: John Wiley Sons

[7] Terrence Fong, Illah R. Nourbakhsh, Kerstin Dautenhahn: A survey of socially interactive robots. Robotics and Autonomous Systems 42(3-4): $143-166$ (2003)

[8] Joan Greenbaum and Morten Kyng (Eds.), Design at Work: Cooperative Design of Computer Systems, Lawrence Erlbaum Associates, Inc., Hillsdale, NJ, 1991.

[9] Takayuki Kanda, Takayuki Hirano, Daniel Eaton, and Hiroshi Ishiguro. 2004. Interactive robots as social partners and peer tutors for children: a field trial. Hum.-Comput. Interact. 19, 1 (June 2004), 61-84.

[10] Iolanda Leite, Carlos Martinho, Ana Paiva, Social Robots for Long-Term Interaction: A Survey. I. J. Social Robotics 5(2): 291-308 (2013)

[11] Mechelen, Maarten Van and Vero Vanden Abeele. Co-design revisited: exploring problematic co-design dynamics in kids. Workshop on Methods of Working with Teenagers in Interaction Design, CHI 2013.

[12] Marvin Minsky. 1974. A Framework for Representing Knowledge. Technical Report. Massachusetts Institute of Technology, Cambridge, MA, USA.

[13] Ahmad, Muneeb, Mubin, Omar, A Orlando, Joanne, A Systematic Review of Adaptivity in Human-Robot Interaction, 2017, J Multimodal Technologies and Interaction, V 1, N 3, P 14

[14] A Paiva, S Mascarenhas, S Petisca, F Correia, P Alves-Oliveira, Towards more humane machines: Creating emotional social robots, New Interdisciplinary Landscapes in Morality and Emotion, 125-139

[15] Papert, S. (1986). Constructionism : A new opportunity for elementary science education. Massachusetts, MA : Massachusetts Institute of Technology, Media Laboratory, Epistemology and Learning Group.

[16] Piaget, J., Inhelder, B., The Psychology of the Child (New York: Basic Books, 1962)

[17] Schank, R.C. (1975). Conceptual Information Processing. New York: Elsevier.

[18] Gavin Sim and Matthew Horton. 2012. Investigating children's opinions of games: Fun Toolkit vs. This or That. In Proceedings of the 11th International Conference on Interaction Design and Children (IDC '12). ACM, New York, NY, USA, 70-77.

[19] A. Strauss and J. Corbin, Basics of qualitative research: grounded theory procedures and techniques, Sage Publications, Newbury Park, Calif, 1990

[20] Marco Botta, Daniele Camilleri, Federica Cena, Francesco Di Sario, Cristina Gena, Giuseppe Ignone, Claudio Mattutino,Cloud-based user modeling for social robots: a first attempt. In the CEUR proceedings of the Workshop on Adapted intEraction with SociAl Robots (cAESAR),IUI 2020

[21] Ahmad, Muneeb and Mubin, Omar and Shahid, Suleman and Orlando, Joanne, Robot's adaptive emotional feedback sustains children's social engagement and promotes their vocabulary learning: a long-term childrobot interaction study, Adaptive Behavior, 2019.

[22] Breazeal C., Takanishi A., Kobayashi T. (2008) Social Robots that Interact with People. In: Siciliano B., Khatib O. (eds) Springer Handbook of Robotics. Springer, Berlin, Heidelberg.

[23] Fong, Terrence and Nourbakhsh, Illah Dautenhahn, Kerstin. (2003). A Survey of Socially Interactive Robots. Robotics and Autonomous Systems. 42. 143-166.

[24] Ahmad, Muneeb and Mubin, Omar and Orlando, Joanne. (2016). Understanding Behaviours and Roles for Social and Adaptive Robots In Education: Teacher's Perspective. 297-304.

[25] VYGOTSKY, L. S.Mind in Society: Development of Higher Psychological Processes. Edited by Michael Cole et al., Harvard University Press, 1978.

[26] Rieber, L.P. Seriously considering play: Designing interactive learning environments based on the blending of microworlds, simulations, and games.ETRD44,4358 (1996).

[27] Jeannette M. Wing. 2006. Computational thinking. Commun. ACM 49, 3 (March 2006), 3335 